\section{Using quotations from non-English interviews in accounting research}

\author{
Sina K. Feldermann
}

University of Siegen, Siegen, Germany, and

\author{
Martin R.W. Hiebl \\ University of Siegen, Siegen, Germany and Johannes Kepler University Linz, \\ Linz, Austria
}

Non-English interviews in accounting research

Received 15 August 2018 Revised 18February 2019 16 August 2019 6 November 2019 Accepted 12 November 2019

\begin{abstract}
Purpose - This paper aims to examine the current practice of reporting on translation issues in qualitative, interdisciplinary accounting research. Based on an analysis of the methodological consideration of the translation of quotations from non-English interviews and additional interviews with experienced researchers, the authors aim to develop recommendations for the reporting on such translation procedures in future accounting research relying on interviews not conducted in English.
\end{abstract}

Design/methodology/approach - The analysis is based on papers published in four highly ranked interdisciplinary accounting journals: Accounting, Auditing and Accountability Journal (AAAJ), Accounting, Organizations and Society (AOS), Critical Perspectives on Accounting (CPA) and Qualitative Research in Accounting and Management $(Q R A M)$. The subjects of the analysis are publications of non-English-speaking researchers who conducted non-English interviews and therefore were confronted with translation issues when attempting to get published in these English-language journals. Additionally, to gain deeper insights into reporting decisions on language and translation issues, the authors conducted interviews with experienced researchers in the field of qualitative, interdisciplinary accounting research whose mother tongue is not English. The authors combine these empirical insights with current developments in translation studies.

Findings - As suggested by translation studies, translation is an act of sense making and reconstruction of meaning, and therefore is a complex task that needs to be carried out with caution. However, the findings suggest that in current interdisciplinary, qualitative accounting research, the reporting of language and translation issues, especially with regards to the translation of quotations from interview data, have so far received only limited attention. The authors therefore call for more awareness of and sensibility toward dealing with language and translation issues, which should be reflected in more transparent reporting on translation processes to support the credibility and authenticity of qualitative accounting studies based on non-English interviews.

Research limitations/implications - This paper is limited to the reporting on the methodological consideration of translating quotations from non-English interviews in papers published in $A A A J, A O S, C P A$ and $Q R A M$ between 2004 and 2015. For future accounting research that relies on such interviews, the authors call for more transparency and provide specific recommendations. This in turn should strengthen the awareness that language and translation are factors to be considered and reported.

(C) Sina K. Feldermann and Martin R.W. Hiebl. Published by Emerald Publishing Limited. This article is published under the Creative Commons Attribution (CC BY 4.0) licence. Anyone may reproduce, distribute, translate and create derivative works of this article (for both commercial and noncommercial purposes), subject to full attribution to the original publication and authors. The full terms of this licence may be seen at http://creativecommons.org/licences/by/4.0/legalcode

A prior version of this paper has been presented at the 15th Annual Conference for Management Accounting Research (ACMAR) in Vallendar, Germany. We acknowledge highly valuable comments by participants at this conference and by two anonymous reviewers which have greatly contributed in improving the initial versions of this paper. We are also grateful to the senior accounting researchers who shared with us their views on the subject in our interviews with them.

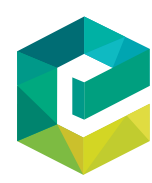

Qualitative Research in Accounting \& Management Vol. 17 No. 2,2020 pp. $229-262$ Emerald Publishing Limited DOI 10.1108/QRAM-08-2018-0059 
QRAM 17,2

Originality/value - This paper is the first to develop recommendations for the reporting of translation processes in accounting research studies, which are based on interviews not led in the English language.

Keywords Accounting research, Language, Interviews, Translation, Quotations

Paper type Research paper

\section{Introduction}

This paper studies the reporting on translation issues in qualitative, interdisciplinary accounting research. Such translation issues may arise, as the most highly ranked international academic journals in accounting research are all published in English, which can be seen as the lingua franca in such research (Enzenhofer and Resch, 2011; Evans and Kamla, 2018). Many non-English-speaking academics are increasingly confronted with the challenge of translating their research results into English to publish them in highly ranked journals, and such translation processes may come with additional language challenges and issues. As put by Humphrey and Gendron (2015, p. 53), the "language issue can be especially problematic for people whose writing skills in English are not as developed as in their native language." As a consequence, there arises the pressure for non-English-speaking researchers and academics to deliver manuscripts written in professional and academic English[1].

The difficulties inherent in language and translation may lead to an exacerbating risk of misunderstanding. As put by Evans (2004, p. 210), "the choice of an inappropriate label in the translation" may be "detrimental to international accounting communication" (see also Kettunen, 2017). Similarly, Komori (2015, p. 142) argues that "[w]hen the researcher's cultural/linguistic background and experiences differ from those of their audience, there is always the risk that their views will be misinterpreted." For example, the German term vorsicht could be translated into English in many ways such as "attention," "carefulness," "caution," "circumspection," "prudence" and "conservatism' (Evans et al., 2015). As argued by Evans et al. (2015), only the last two translations would fit an accounting context and thus present a proper translation, as vorsicht is regarded as a principle of prudence in the Germanic accounting context. Such language and translation issues are particularly important in qualitative interview-based research. Interviews often aim to unveil interviewees' subjective experiences, usually expressed in their source language - that is, for the rest of this paper, a language other than English.

However, in many cases, the methodological consideration and translation of qualitative data are uncertain (Evans, 2016; Kruse, 2012; Xian, 2008). Researchers in disciplines other than accounting such as medicine, psychiatry and psychology acknowledge and report on the problems associated with translations as shown by the extensive methodological literature on translation-related issues in these disciplines (Evans et al., 2015). In particular, the importance of the translation of quotations and reporting on such issues seem more advanced. In contrast, Evans et al. (2015, pp. 2-3) show, "that the importance of translation in accounting is underestimated or disregarded," which is reflected in "little examination and exploration of translation problems in accounting research." Especially for "qualitative, interdisciplinary accounting research", Evans and Kamla (2018, p. 1834) argue that the "silence on language and translation is particularly surprising" as it:

[...] has a long-standing tradition of acknowledging the instrumental role played by language in society, and of challenging mainstream market-based research for neutralizing cultures and ignoring the subjective role of the researcher in the process.

In qualitative, interview-based accounting studies, we can assume that translation problems primarily materialize in direct quotations used in the manuscripts reporting on research 
results[2]. For such quotations, researchers need to translate material directly from nonEnglish interviews into the English language, while for the rest of their results, such direct translation is not normally needed. In general, direct quotations from interviews are seen as an opportunity to achieve credibility and authenticity in qualitative accounting studies. Such quotations are therefore an important quality criterion for qualitative studies (Messner et al., 2017). However, a proper translation of quotations from non-English interviews may not be easy to achieve, since the original meaning of the quotations needs to be preserved. For translated quotations from non-English interviews, suitable and transparent processes may thus be necessary to ensure the quality of translations and to demonstrate that special care has been taken when translating interview quotations. Adequate reporting on such processes may eventually support the credibility and authenticity of qualitative accounting studies, which are usually required for publication in highly ranked accounting journals (Messner et al., 2017; Scapens, 2004). So while quotations play an important role in qualitative accounting research, their selection and interpretation is still surrounded "by a degree of mystery" (Dai et al., 2019, p. 29). While such mystery may not be entirely removed from qualitative accounting research (Dai et al., 2019), we argue - as detailed below - that more awareness of and sensibility toward the translation of quotations is needed. This increased awareness and sensibility could be reflected in more transparent reporting on the translation of interview quotations, which in turn may raise the credibility and authenticity of qualitative accounting studies. Our focus in this paper therefore rests on reporting how quotations from non-English interviews are translated into English.

Note that this focus of our paper should not imply that we question or even distrust quotations from non-English interviews published in prior accounting research. Based on our analyses, we rather see room for even more credible and authentic studies fueled by more transparent reporting on the translation of interview quotations. We do, however, not see increased transparency as a desirable end in itself. We rather envision that researchers may need to increase their awareness of and sensibility toward translating interview quotations to report them more transparently. That is, if researchers follow our recommendations on more transparent reporting of translating interview quotations, they are induced to critically reflect on their interview translation procedures, which may in turn increase their awareness of and sensibility toward this part of the research process. In addition, more transparent reporting may contribute to the credibility and authenticity of the study at hand.

Given the importance of quotations and reporting on translation processes in interviewbased, qualitative accounting studies, we have two specific objectives in this paper. First, we analyze the status quo of the reporting on translation processes for quotations from nonEnglish interviews in studies published in four highly ranked interdisciplinary accounting journals. These four journals are Accounting, Auditing and Accountability Journal (AAAJ), Accounting, Organizations and Society (AOS), Critical Perspectives on Accounting (CPA) and Qualitative Research in Accounting and Management (QRAM). We analyze articles published in these four journals between 2004 and 2015 and which are authored by nonnative English-language researchers who collected data in a language other than English. Furthermore, to gain deeper insights into the relevance of language and translation issues and the reporting on them, we conducted interviews with experienced researchers in qualitative, interdisciplinary accounting research whose mother tongue is not English. Following these exercises, and by drawing on insights from translation studies, our second objective is to develop recommendations for reporting on the translation of interview quotations for future accounting research relying on non-English interviews. To this end, we derive specific recommendations that we offer at the end of this paper.
Non-English interviews in accounting research 
QRAM 17,2

The rest of this paper is structured as follows. The next section provides an overview of the relevant background and introduces the literature on interview data and translation issues. We also point out the role of interviews and quotations from interviews in achieving credibility and authenticity in qualitative studies, and to this end, we draw on insights from translation studies and linguistics. The following section briefly reviews the consideration of language and translation in research in general, and in accounting research more specifically, especially with regards to interviews. After the presentation of our research methods, we report on our findings and provide a critical discussion. Based on the preceding analyses and findings from translation studies, we then develop recommendations on the reporting of translation processes in future accounting research relying on non-English interviews. We conclude the paper with the most important implications arising from our analyses.

\section{Interview quotations and translation issues in the research process Credibility and authenticity of interview data and quotations}

In qualitative research, interviews are often used as the main data collection method (King, 2006), as they allow researchers to:

[...] examine social phenomena, not by transforming them in quantitative terms, but by confronting them on their own naturalistic terms, so that they reveal the meaning of practitioners assign to actions and events as they act (Covaleski et al., 2017, p. 392).

Interviews are used "to produce rich accounts that capture and communicate the complexity of the social and organizational world" (Messner et al., 2017, p. 432). Qualitative data gathered through interviews thus aim to capture special aspects of social knowledge or the subjective experiential reality of interviewees (Condie, 2012; Qu and Dumay, 2011). To this end, quotations from interviews seem very relevant since "they provide a sense of meaningfulness to the interpretive claims advanced by the authors" (Dai et al., 2019, p. 29). The usage of quotations in research studies thus allows the researcher to present the subtleties and nuances of a situation (Marschan-Piekkari and Reis, 2009) and to enable the reader "to experience what it would be like to hear, see, or feel what the original speaker [the interviewee] did" (Wade and Clark, 1993, p. 818). For this reason, quotations could be seen as an act of multimodal (re-) construction of what has been said in a specific way in a specific situation (Blackwell et al., 2015). This is also true in accounting research (Qu and Dumay, 2011; Ryan et al., 2002), and thus qualitative accounting research is "not judged by the "quantity" of examples provided"-for instance, quotes from interviews, excerpts from meeting discussions and copies of company documents-"but by their strength and significance" (Messner et al., 2017, p. 438).

Data quality in interview-based studies can thus be interpreted as a key criterion for the overall quality of the underlying research. To make such research quality more tangible, Messner et al. (2017), Lukka and Kasanen (1995) and Parker (2012) suggest the concepts of credibility and authenticity for qualitative research[3]. These concepts are meant as alternatives to "validity" and "objectivity" focused upon in quantitative studies. Credibility refers "to the extent to which a qualitative account is convincing in terms of its proposed findings" (Messner et al., 2017, p. 433), while a study is considered authentic "if it skillfully exploits the richness of the empirical material rather than providing only highly condensed findings as in the form of abstract theoretical propositions or the like" (Messner et al., 2017, pp. 436-437; see also Golden-Biddle et al., 2006). The achievement of authenticity can support qualitative research by two means. First, by supporting the credibility of the findings and second, by materializing in an effective communication of research results to readers: 
authentic writing captures the "complexity of the field" and provides "the reader with the phenomenological detail that is necessary to really "grasp" the complexity" (Messner et al., 2017, p. 437).

Direct quotations from interviews are a primary opportunity to present the collected data in an authentic way, as it is assumed that the reader's assessment of the authenticity, reliability and plausibility of the study might rely heavily on the data they actually see (Messner et al., 2017; Pugh and Vetere, 2009). In a qualitative research manuscript, readers often only see a small percentage of the underlying empirical data in the form of quotations from the interviews (Aguinis and Solarino, 2019; Dai et al., 2019). As argued by Pratt (2009), in many cases researchers will choose the quotations that are most "powerful", meaning:

[...] those that illuminate a point that the researcher wishes to make particularly well and that often feature vivid examples and colorful expressions, such that these quotes would be difficult to translate into indirect ones without losing much of their appeal (Messner et al., 2017, pp. 438-439).

If the interview and the intended quotation were conducted in a language other than the target language of publication (i.e. in the case of this paper, English), the researcher is confronted with translation problems such as ensuring that the subtleties and nuances expressed by the interviewee are captured by the translated quotation. As stated by Humphrey and Gendron (2015), accounting texts, representations and data that are developed and collected in one language may not be reproduced "perfectly" in another language, which also applies to quotations from interview data. Consequently, to support the credibility and authenticity of qualitative research studies, a proper translation of quotations from interviews can play a decisive role. We do not argue that prior accounting researchers would not have provided such proper translations, but just as with any methodological choice that remains unexplained, readers - and in particular, reviewers - of qualitative research papers cannot assess the extent to which the methodological steps taken are appropriate for the intended purpose. That is, for readers and reviewers, it is often difficult to assess whether a proper and authentic translation has been conducted because they are usually only presented with the translated quotations in English and not with the original quotations in the source language. In many cases, readers and reviewers will therefore have to rely on the translation offered by the authors of the manuscript. However, as explained by Evans et al. (2015), obvious errors in translations could result in a loss of credibility. Aguinis and Solarino (2019) go even further by arguing that a lack of transparency could affect the trustworthiness of the published research. In their review of the transparency and replicability of qualitative studies relying on interviews with elite informants in strategic management research, they conclude that none of the 52 articles analyzed is fully transparent in explaining what has been done, why and how. Consequently, transparently reporting on translation processes in general and the quotations used may offer authors an opportunity to demonstrate that they are aware of and sensible toward potential translation issues and have taken sufficient measures to preserve the original source-language meaning of their interview quotations, which in turn should support the credibility and authenticity of their entire study. The preservation of meaning in interview data, however, is a non-trivial task. The issues potentially arising from such translations will be explained in the following, relying on insights from translation studies and linguistics.

\section{Some implications from translation studies, linguistics and other disciplines for translating interview data}

According to Gambier (2004), translation is located in a field consisting of complexity and multidisciplinarity and is related to a variety of problems that need to be considered during the research process. Following Schäffner (2004), translation problems may arise, for 
QRAM 17,2

instance, in terms of linguistic aspects, textual and discursive features, and with regard to situational, cultural or sociological issues. Whereas linguistic aspects refer to issues such as equivalent structures between source and target language, textual and discursive features refer to aspects such as text types, genres or text functions, while the other issues refer to the impact of translation on cultural developments, amongst other things.

Creating equivalent structures is a core task in translation processes (Enzenhofer and Resch, 2011). For instance, the translation of the bible or a poem may require the reconstruction of syntax, lexis, and style in the target text. As explained by Enzenhofer and Resch (2011), this concept of equivalence refers to the establishment of similarity between the source and the target languages at the textual level. Therefore, translations using such concepts may not deviate significantly on the formal level as compared with other approaches. In some cases, it does not make sense to create equivalence - for instance, when the audience of the source and target languages/texts differ significantly. This situation might be the case when texts (or something being said in an interview) break religious or sexual taboos - for example, when translating advertising texts from the American to the Arab world (Enzenhofer and Resch, 2011; for an overview see also Evans et al., 2015). Creating equivalence in such cases might not be the ideal practice for translation, so a functionalist approach seems to be more suitable. The functionalist approach of translation aims for the achievement of a translation initiator's needs (Schäffner, 2009), which may involve ensuring the target text is understandable for an end user of, say, an advertisement or a technical instruction manual. Consequently, the translated text may break away from the original text[4].

The assumption that data can be translated by the simple translation of words refers to the positivist assumption of an obtainable reality independent of the actors, languages and social contexts involved. This understanding implies that words and meanings are equivalent in different languages. However, Simon (1997) notes that words are not equivalent in different languages and that language carries a cultural meaning. As indicated by the example of the German term vorsicht above, concepts and themes are not necessarily equivalent across cultures and languages (Xian, 2008). As a consequence, Xian (2008, p. 232) argues that there is no "obtainable 'reality' between languages through translation." This problem is exacerbated for translation and dissemination of research findings from nonAnglo-Saxon study settings to an audience that is shaped by anglocentricity. Such dissemination requires both the translation and the interpretation of indigenous phenomena, knowledge, and concepts to a non-indigenous language - that is, English (Komori, 2015). These indigenous phenomena might be unknown in the international anglocentric research community. Consequently, such non-Anglo-Saxon phenomena may suffer from miscommunication and misinterpretation (Komori, 2015), particularly when considering that the risk of misinterpretation even occurs when translating and disseminating research results across similar cultural contexts (Evans, 2004; Evans et al., 2015).

Translation could be seen as an act of mediated communication (Schäffner, 2004) and is characterized as:

[...] offering information to members of one culture in their language (the target language and culture) about information originally offered in another language within another culture (the source language and culture) (Schäffner, 2009, pp. 117-118).

Given these issues, translation ideally needs to be understood as a process characterized by comprehension, sense making and the re-expression of ideas (Salama-Carr, 2009; Simon, 1997). For example, Temple (1997, p. 609) argues that "language at the same time constructs as it describes the world. Words depend for their meaning on the circumstances of their 
production." The challenge for the translator and researcher is not only to translate "the linguistic meaning of the source language," but also to reconstruct "the meaning of the source language" and convey it to the readers of the translation (Salama-Carr, 2009, p. 145). Harkness (2003, p. 48) defines what "meaning” may actually mean:

The four components central to clarifying what is meant with a given utterance in a given context are what is said, what the speaker's intentions are (what the speaker/writer intends to be understood), the 'common ground' between participants (the shared information participants in an interaction have [...]), and the macro and micro contextual setting of an utterance.

According to Xian (2008), a successful translation comprises the transfer of the original meaning and does not mean the preservation of the original linguistic form in any case[5]. Thus, we can conclude that if translation is the interpretation and reconstruction of meaning, there is no one "right" interpretation and no one "right" reconstruction. Translation can only be an approximation of the meaning from the source language in the target language. Consequently, for the translator it might be all about finding a translation of interview data with which he or she is comfortable in terms of achieving this aim (Pentland, 1993).

When translating quotations from interviews for usage in publications and thus for the purpose of this paper, achieving this aim means ensuring the transfer of meaning of what has been said (Harkness, 2003), while reconstructing the colorful expressions (Messner et al., 2017), the nuances and the subtleties (Marschan-Piekkari and Reis, 2009) of the original quotations. This aim of translating non-English quotations could support the credibility and authenticity of qualitative studies (Messner et al., 2017), whereas it should ensure that the generated knowledge is not lost in the process of translating it for publication (Liyanage et al., 2009).

In addition to the mentioned challenge of an authentic translation, two further aspects should be considered when translating quotations from qualitative interviews: the role of the researcher as the author and translator, and the role of the audience for which the translation is intended. As pointed out by Gile (2004, p. 125), who argues for more interdisciplinarity between translation research and other disciplines (e.g. accounting), the understanding of the "human factors" play an important role. That is why Holland and Ramazanoglu (1994, p. 132) argue that "differences such as age, class, gender, ethnicity and religion impinge on the possibilities of interaction and interpretation, and so on how the social world is known." In addition, as pointed out by Dai et al. (2019, p. 3), "social norms play a significant role in people's sense-making". This applies not only to the interviewee, but also to the interviewer and translator of a quotation, whose social norms influence the sense making while reconstructing the meaning of what has been said. The act of translation is therefore influenced by the individual who carries out the translation and his or her own worldview and interpretation of the source language, culture and meaning (Temple, 2008; Temple and Young, 2004). As pointed out by Xian (2008), the translator interacts with the data while actively interpreting their implicit social concepts and meanings.

As indicated above, when translating quotations from interviews as an act of representation, caution is required since there is a difference between:

- simply translating interview data by reconstructing the words; and

- the translated interview data as the outcome of a reconstruction of meaning.

Something said in the source language and the underlying styles and themes may get lost while reconstructing them in the target language, especially when concepts and themes are well known in the target culture, but unknown in the source culture. As a consequence, this
Non-English interviews in accounting research

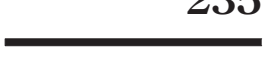


QRAM 17,2

could lead to the conformity of data and "to styles and themes that currently prevail in the target culture" (Xian, 2008, p. 237). For this reason, as highlighted by Blenkinsopp and Pajouh (2010), the risk of getting "lost in translation" refers to the loss of connotations and cultural salience and - in the worst case - the loss of meaning. Evans et al. (2015) refer to this as the risk of misunderstanding and inappropriate labeling.

So far, we can conclude that the aim for the translation of non-English interviews as an act of sense-making and interpretation might be the authentic reconstruction and recreation of the original meaning, and that "meaning" is defined inter alia in terms of the macro and micro contextual setting of the original interview (Harkness, 2003).

\section{Language and translation in methodological and accounting literature}

In international, cross-cultural and comparative research - especially in quantitative research - the role of language and translation is one of the most frequently mentioned problems (Harkness, 2003). A considerable amount of studies has been published on diverse aspects of translation, including research or data collection instruments such as survey questionnaires.

For example, Harkness (2003) discusses the practical implementation and assessment of questionnaire translations. The author argues that translators, translation reviewers, and translation adjudicators are necessary to translate a survey. Considering that translation is not just a matter of finding the right words, Harkness (2003, p. 41) states that back translation "is the best known translation assessment procedure in survey research" for producing a high-quality translation (van de Vijver and Tanzer, 2004). This method, originally developed by Brislin (1970), constitutes a framework that first requires the translation of the source language into the target language by an interpreter, and thereafter a back translation from this translated target language by another independent interpreter into the source language. By comparing the original version with the back-translated version, the accuracy of the translation can be assessed. In turn, Welch and Piekkari (2006) explore the use of foreign languages in qualitative interviewing in English-dominated international business research. They show that researchers have to make multiple decisions about the language they use (project language, access language, interview language, and post-interview language) and the effect it has on data accuracy, credibility, and authenticity.

These examples indicate that the challenges posed by diverse languages and translation issues are not new to the methodological literature, which has shown that translation issues are often present in the early phases of research studies - for instance, when designing and testing questionnaires. As Marschan-Piekkari and Reis (2009, pp. 223-224) point out, "[o]nce it has been finalized and the data collection has commenced in different countries, the language issue is considered "solved" and its implications tend to be forgotten". Consequently, the methodological literature lacks recommendations for dealing with language and translation issues in the later stages of the research process, such as the final publication of research findings, which - in the case of interview-based studies - may include quotations from interviews.

These findings also broadly apply to accounting research. Although no extensive body of the literature in accounting research deals with translation issues, prior research indicates that translation issues are experienced by some researchers. A part of this literature deals with various accounting concepts and their different perceptions by different interest groups. For example, the research by Oliver (1974) and Houghton and Hronsky (1993) shows that different stakeholders, due to their cultural background and language (i.e. their specific contextual settings), perceive and interpret accounting concepts in different ways. 
Further research focuses on the translation of accounting terminology, technical terms and accounting principles. For instance, the study by Bagranoff et al. (1994, p. 35) provides evidence that "cross-cultural differences may affect the meaning of, and hence the application of, accounting principles." In a case study, Evans (2004) examines the different interpretations and perceptions of technical accounting terms such as the German "grundsätze ordnungsgemäßer buchführung," or the "true and fair view," and the problems that arise when translating them into other linguistic and cultural areas. With regards to the translation of technical accounting terms and concepts, researchers seem to be especially aware of the relevance of translation and translation issues. For instance, studies by Alexander (1993), Nobes (1993, 2009), Doupnik and Richter (2003), and Baskerville and Evans (2011) demonstrate this awareness, as all of these researchers examine the translation and implementation issues of selected accounting terms such as the "true and fair view" or uncertainty expressions about EU accounting directives.

Further studies compare the methodological consideration of translation issues in different disciplines. For example, Evans et al. (2015, p. 2) compare the handling of translation issues in accounting research with their handling in other disciplines, such as law and medicine, and conclude that in comparison with other research disciplines, "there is, to date, little examination and exploration of translation problems in accounting research." Similarly, Kamla and Komori (2018, p. 1877) argue that there is a "disconcerting silence" on language and translation issues in accounting research. With respect to research in management and organization, Steyaert and Janssens (2012) even go so far as to say that the use of English in academia seems to be unreflected upon.

Although translation practices and concerns may vary across disciplines, "there are also many shared problems, and there is considerable overlap in approaches and solutions" (Evans et al., 2015, p. 2). Consequently, the treatment of language and translation issues in disciplines other than accounting could inform accounting research. For example, Santos et al. (2013a, 2013b) provide a good example of a closer consideration of the translation of interview quotations. While examining the experience of postpartum depression in Brazilian women, the authors became aware of the difficulties inherent in the translation of interview data and quotations for publication. Indeed, the description of the translation process of interview data and of the involved translators/interpreters and their experiences is extensive (Santos et al., 2013a, 2013b). Given the importance of translating and preparing interview quotes for publication, their follow-up article addressed how to deal with translation issues (Santos et al., 2015). They provide examples of the difficulties experienced by using two possible translations for the original Portuguese quote "Eu estava aterrorizada com [...]" They discuss the following two translation alternatives suggested by an interpreter and provided by themselves, respectively: "I had panic attacks about [...]" and "I was terrified about [... ." The authors state that the first translation, which appears to be a type of medical issue, is a misinterpretation because of the inaccurate translation, as this statement is marked more by emotions than a medical understanding in the source language. Another example, "Eu tive muitos pensamentos ruins," could be translated as either "I had very bad thoughts," which focuses on the intensity of one's thoughts, or "I had many bad thoughts," which focuses on the quantity of bad thoughts (Santos et al., 2015). For these authors, only the latter option constitutes a "comfortable" (Pentland, 1993) translation. These examples show that the meaning and sense of what has been said can vary significantly in different translations by different translators even though the available translations are similar at first glance[6]. These findings from disciplines such as medicine, psychiatry and psychology confirm the conclusion by Evans et al. (2015, p. 13) that "the importance of translation in 
QRAM

17,2

medical disciplines is [...] reflected in a much more organized response to its problems than is the case in other disciplines".

We therefore conclude that accounting research related to language issues has mostly concentrated on technical accounting terms. While other disciplines have shown more consideration of such issues (Santos et al., 2015), accounting research has paid particularly little attention to the translation issues arising from qualitative interview-based studies. Further, the international accounting literature has mostly neglected input from translation studies and linguistics, as detailed above. Dai et al. (2019, p. 10), based on a broad review of interview-based accounting research, go even further and conclude that they are "concerned with the ways in which interview data is articulated and mobilized in research articles." The present study aims to address a part of these concerns for the specific case of reporting on the translation of quotations from non-English interviews.

\section{Research methods}

General research approach

To address the research objectives defined above, we rely on three sources:

(1) an analysis of prior interview-based accounting studies published in $A A A J, A O S$, $C P A$ and $Q R A M$;

(2) interviews with seven experienced accounting researchers who have published at least one paper each in our sample of reviewed studies; and

(3) recent insights from translation studies for developing some recommendations for future accounting research.

While we performed the literature analysis first and then led the interviews, some of our methodological choices could be confirmed thanks to the results of the interviews with the experienced accounting researchers and thus reinforced our methodological choices.

\section{Literature analysis}

Similar to Parker and Northcott (2016), our analysis is based on four leading interdisciplinary accounting journals: $A A A J, A O S, C P A$ and $Q R A M$. These journals were chosen because they are frequent outlets of qualitative accounting research (Hiebl, 2018; Parker and Northcott, 2016)[7]. In turn, in qualitative accounting research, interviews appear to be the most frequently used data collection method (Ryan et al., 2002). Thus, AAAJ, AOS, $C P A$ and $Q R A M$ can be expected to feature a significant number of studies relying on interviews.

Subject to the present paper is the methodological consideration of translation issues in the selected articles, especially with regard to translations of quotations from non-English interviews. We therefore focus on articles where we can assume that translation issues existed, which in our case means that research, notably interviews, was carried out in a language that differs from the language of publication, which is English in the case of $A A A J, A O S, C P A$ and $Q R A M$. Therefore, we aim to include studies in our analysis of which we can assume that translations were part of their research process. To identify articles where translations have been part of the process is a tricky task, as this is an issue that remains mostly unreported upon in the published articles (as our below findings show). In consequence, we had to resort to other opportunities of identifying research articles where translation issues in the underlying research process are likely to have been present. To this end, we decided to use the affiliations of the authors of the published papers as an approximation for a high probability of arising translation issues. 
Hence, to select relevant studies, we used the official language of the country of the author's affiliation, as published in the found articles, as a proxy for arising translation issues. Our underlying assumption (A) is the following:

A1. We assume that the interviews in the analyzed studies were conducted in the country of the author's affiliation. We further assume that the interviews were conducted in the official language of the country in which the author's institution is located.

Non-English interviews in accounting research

This assumption was confirmed in our interviews with seven experienced scholars (see below for more details). They all indicated that in their studies, those that we have included in our sample, the data collection took place in the country of their affiliation. Furthermore, all interviewees explicitly mentioned that interviews were conducted in the official language of the country of the authors' affiliation. Following assumption A1 and our preliminary analyses[8], articles written by authors who work at an institution located in a country whose official language is English are excluded from the dataset, as it is supposed that no translation issues related to English-language publications existed. We acknowledge that this - and the following - approximation involves some limitations, which are acknowledged in the following subsection.

With the help of assumption A1, we can classify publications authored by one author as being either relevant or non-relevant for this study. For author teams - that is, a group of at least two authors - we need further assumptions. In line with A1, we assume:

A2. For author teams where all authors were affiliated with institutions in countries whose official language is English, we assume that the underlying interviews were conducted in English and that no translation issues arose.

A3. For author teams where all authors were affiliated with institutions in countries whose official language is not English, we assume that the underlying interviews were conducted in a language other than English and that translation issues arose.

There remain, however, mixed-language author teams. For our purposes, we define a mixedlanguage author team as a group of at least two authors who work at institutions located in countries with different official languages, where at least one of these languages is English. For instance, a mixed-language author team could be made up of one Australian author and one Italian author. As our preliminary analyses had shown that reporting on translation issues is weak in most studies, we could not find a clear proxy for identifying articles from mixed-language author teams in which translation issues arose. That is, for our example of the Australian-Italian author team, the underlying interviews are likely to have been conducted in English in Australia or have been conducted in Italian in Italy[9]. Consequently, we had the choice either to include all articles by mixed-language author teams or to completely exclude them from our sample. As articles by mixed-author teams may include valuable information on translation issues and how authors dealt with them, we decided to include preliminarily articles by such teams. By making this choice, we deliberately accepted that our preliminary sample might include cases in which interviews were conducted in the English language. That is, for our example of the Australian-Italian author team, the interviews might have been conducted in English, and thus no translation issues would have arisen, but the article would nevertheless be included in our sample since such articles mostly do not include information on the language of data collection.

Special cases are articles by Canadian authors, which frequently feature in the four journals that we analyze. While Canada has English as one of its official languages, 
QRAM 17,2

significant parts of the population - most notably the population in the province of Quebec primarily speak French (Corbeil and Blaser, 2007). In those parts of the country, it can be assumed that many research interviews are conducted in French. The sole reliance on the authors' affiliation country, in line with A1, would have led to the exclusion of all studies by authors with affiliations in Canada. As many of these Canadian studies are authored by scholars with Francophone names, we can assume that these authors frequently conduct their research interviews in French. Consequently, we decided to include preliminarily studies authored or co-authored by Canadian authors.

Following these assumptions and choices, we preliminarily included articles which:

- were written by authors whose institution(s), as declared in the published articles, are located in a country whose official language is not English or is in Canada; or

- were written by a mixed-language author team where at least one of the authors works at an institution located in a country whose official language is not English or is in Canada.

In these two cases, translation issues relevant to our research objectives could arise. Relevant articles in terms of translating non-English interviews were identified by using a keyword search in the online databases of $A A A J, A O S, C P A$ and QRAM[10]. For the preliminary data selection, the search included the following keywords: Interview, interview*. We limited our search to articles published between 2004 and 2015, as this period was considered to be long enough to identify trends and to allow for insights into more recent research practice (Wagenhofer, 2006).

Conducting the keyword search while considering the above assumptions led to a preliminary sample of 392 articles that were subject to an in-depth examination to identify translation issues and their methodological consideration. First, the articles' abstracts, method sections, acknowledgements, and notes were carefully read to identify annotations with regards to translation issues[11]. Furthermore, the search function of Adobe Acrobat was used to ensure that all relevant passages were found. Searches were conducted by using the terms "interview," "translation," "language" and "English." As part of this process, 146 articles were excluded from our further analyses, the main reasons being:

- The authors did not conduct interviews. Such articles were found in our initial keyword search since the authors refer to interviews, for instance, in the literature review sections (exclusion of 126 articles).

- The interviews were conducted in a country whose official language is English or it was explicitly mentioned that the interviews were conducted in English. In consequence, translation issues did not arise and the articles were excluded from the preliminary sample (exclusion of 20 articles).

As a result of the keyword search, our study includes articles of different methodological approaches. Therefore, we examine articles that rely only or primarily on interviews as well as mixed-method studies, in which interviews were conducted to capture additional information, besides other data-collection methods such as surveys.

Of the 246 articles remaining after this step, 68 were published in $A A A J, 76$ in AOS, 56 in $C P A$ and 46 in QRAM. A total of 128 articles were written by authors whose institution is located in a country whose official language is not English, while 118 articles were written by mixed-language author teams where at least one of the authors' institution is located in a country whose official language is not English or is Canada. The findings presented below are based on the analyses of these 246 articles included in our final sample and interviews with experienced scholars. 


\section{Interviews with established researchers}

To gain deeper insights into reporting decisions on language and translation issues, we complemented our insights from the literature analysis with seven semi-structured interviews with established researchers[12]. These researchers are all experienced in conducting qualitative interdisciplinary accounting research and their mother tongue is not English, which corresponds with A1. We tried to interview both researchers who reported on translation issues in their studies included in our sample and others who did not report on such language issues. This approach allows us to capture the views and motivations from researchers leading to their reporting or non-reporting on translation issues. To identify "experienced researchers," we relied on their publication record. That is, we addressed researchers who have published multiple times in ABS-ranked journals and of which at least one article has been published in a journal with an ABS grade of three or higher. Most of our interviewees even have publications in journals with an ABS ranking of four or higher. While we are aware that journal rankings such as the ABS guide need to be interpreted with caution (Humphrey and Gendron, 2015; Tourish and Willmott, 2015; Willmott, 2011), we argue that publications in highly ranked and highly regarded journals can be seen as a signal that researchers are experienced. Such rankings were therefore instrumental for our purposes. To include some variance in our group of interviewees, we tried to address interviewees stemming from differing cultures and having different mother tongues, research interests, and topics. Our interview sample consists of researchers whose primary affiliation is located in Austria, Finland, France, Germany, Italy, Romania and Sweden. The interviews were conducted in November and December 2018, lasted about half an hour each and were recorded and transcribed. All interviews were conducted in English because:

- The methodological choices we discussed with the interviewees did not require the usage of very specific and thus translation-sensitive language.

- We do not speak some of the mother tongues of our interviewees and wanted to approach all interviews in the same language to avoid potential language biases between interviews.

- Both the interviewees and the interviewers are experienced in presenting and publishing research in English, which allows for fluent interviews conducted in English.

The interview guide consisted of questions regarding the researchers' choice of the country and the language in which their interviews were conducted, their handling of (potential) translation issues, the translation process, and their specific reporting decisions in general and with regard to the usage of direct quotations. Furthermore, we also asked the interviewees about their views of the importance, advantages, and disadvantages of reporting and not reporting on language and translation issues in their papers and in general.

\section{Development of recommendations}

As indicated above, in the below development of our recommendations for further accounting research, we draw on three data sources:

(1) the insights from our literature analysis;

(2) the interviews with experienced scholars; and

(3) recent insights from translation studies (see our above literature review section).
Non-English interviews in accounting research 
QRAM 17,2

In particular, we compared the current practice of reporting on language and translation issues identified from our literature analysis with the views of experienced scholars and suggestions from translation studies. As will be revealed below, this comparison exposed some significant gaps or inconsistencies between our three data sources. Our recommendations are meant to address these gaps.

\section{Limitations of the chosen research approach}

In particular, our data selection process in the literature analysis is subject to some limitations that should be mentioned to better understand the present study. First, the selection of keywords ("interview"/ "interview*") in our search for relevant studies may be a limitation, as the choice of these keywords may have prevented the identification and incorporation of some articles based on conversations and/or discussions, which could also have featured translated quotations. However, given that interviews are probably the most often used data collection method in qualitative research (Qu and Dumay, 2011), we are confident that we captured the largest part of such research potentially subject to quotation translation issues.

Second, using the official language of the country of the authors' affiliation as the preliminary indicator to identify studies where translation issues might have arisen could be considered an inadequate approach. However, given the unavailability of necessary information to use more elaborate indicators from public sources, and due to the lack of reporting in the included publications, this was the only practicable approach we could identify. Our preliminary analysis reported above and the results from our interviews with experienced accounting researchers (see below) confirmed that - while our approach may not be perfect - we could capture a large part of the respective studies with this approach.

A further limitation is that articles may be included in our literature analysis, even though translation issues might not have existed. On the one hand, articles by authors who worked at an institution located in a country whose official language is not English, but nevertheless conducted interviews in English, could be included in our sample. In this case, translation issues would not have existed. On the other hand, articles by authors who conducted interviews in a language other than English could be excluded, because their published institution is located in a country whose official language is English. In this case, translation issues would have arisen, yet are not analyzed in our paper here. Due to insufficient reporting in many studies, we could not ascertain whether the former or the latter situation might have been the case.

Despite these limitations, we are confident that our relatively large sample of 246 analyzed articles allows for insights into the current treatment of translation issues in interview-based, interdisciplinary accounting research, and their combination with insights from interviews with experienced accounting scholars and translation studies allows for the development of tangible recommendations for future accounting research.

\section{Findings}

Literature analysis

Table I shows the allocation of the 246 articles to the four interdisciplinary accounting journals during the analyzed 12 years. Similar to the study by Dai et al. (2019), it can be seen that the number of publications using interviews as part of their research has risen from 10 publications in 2004 to 33 publications in 2015. This underpins the role of interviewing in qualitative research as an increasingly common method for gathering data (King, 2006; Mcdonald and Hellgren, 2009). 
As indicated above, 118 of the 246 articles were written by a mixed-language author team where at least one of the authors worked at an institution located in a country whose official language is not English or in Canada. In 18 of these 118 articles, the authors refer to language and translation in the research process, while the other 100 articles do not contain any information on language or translation. The remaining 128 articles out of the overall 246 articles were written by authors (or author teams) who were (all) located in countries whose official language is not English or in Canada. Of those 128 articles, only 23 refer to language and translation issues. The other 105 articles give no information on language or translation.

To summarize, of the 246 articles included in our literature analysis sample, only 41 included some information on translation issues. The remaining 205 papers, the vast majority of the whole sample, did not comment on translation issues in any form. Only in eight of the 41 articles including some information on translation did the authors explicitly refer to the translation of the underlying interviews and quotations from these interviews. As Table II indicates, in five of these eight cases (B1, B3, B5, B6, B7), the authors noted the specific language in which the interviews were conducted.

In six cases in our literature analysis (Table II: B1, B2, B3, B5, B6, B7), the authors themselves translated the interview passages used for quotations in the published papers. In two cases (B2, B8), it is only mentioned that the interviews were translated for the quotations, without mentioning the specific source language. For the papers written by authors located in Canada, both cases (B2, B6) revealed that the interviews were not conducted in English. In case B2, the language of translation is not mentioned, although it is obvious that a translation was necessary as the research topic was "french cours des comptes" and a note was included after the quotation to explain that the interviews were conducted in French. For B6, it is noted that the interviews were conducted in French.

All statements on the translation of quotations were found in the methods sections of these papers or in brackets immediately after the specific quotations. Table III provides exemplary findings dealing with further language-related issues beyond translating quotations. These selected findings are comparable with and provide examples for the information that is commonly given in the 41 articles. In 25 of the 41 articles, the authors refer to the language in which the research was conducted and the individuals responsible for the translation. Such papers disclose the language of data collection and analysis as well as the reasons for using the respective languages $(\mathrm{O} 4, \mathrm{O} 5)$ and the handling of specific (not) translated terms and expressions $(\mathrm{O} 1,02)$. Other studies within these 41 articles include thanks to colleagues for revising English-language texts (O3). Although these examples are not directly related to the translation of quotations, they offer some evidence regarding translation issues (e.g. O1) and aspects (e.g. O4, O5) that provide additional value for the following discussion of the findings.

Overall, our findings from the literature analysis seem to be consistent with the observations in our above literature review section that the relevance of translation and related issues is primarily seen during data collection. In all cases, information about

\begin{tabular}{lrrrrrrrrrrrrr}
\hline Journal & 2004 & 2005 & 2006 & 2007 & 2008 & 2009 & 2010 & 2011 & 2012 & 2013 & 2014 & 2015 & Totals \\
\hline AAAJ & 3 & 6 & 3 & 4 & 5 & 6 & 9 & 6 & 7 & 7 & 4 & 8 & 68 \\
AOS & 6 & 4 & 5 & 6 & 6 & 7 & 8 & 10 & 4 & 5 & 8 & 7 & 76 \\
CPA & 1 & 2 & 2 & 2 & 3 & 3 & 7 & 3 & 5 & 7 & 11 & 10 & 56 \\
QRAM & 0 & 1 & 7 & 2 & 3 & 4 & 4 & 2 & 5 & 4 & 6 & 8 & 46 \\
Totals & 10 & 13 & 17 & 14 & 17 & 20 & 28 & 21 & 21 & 23 & 29 & 33 & 246
\end{tabular}

Non-English interviews in accounting research

243 
QRAM

17,2

\section{4}

\begin{tabular}{cll} 
No. & Author (Year) & Title \\
\hline B1 & $\begin{array}{l}\text { Vinnari and } \\
\text { Laine (2013) }\end{array}$ & $\begin{array}{l}\text { Just a passing fad? The } \\
\text { diffusion and decline of } \\
\text { environmental reporting } \\
\text { in the Finnish water } \\
\end{array}$ \\
& & sector
\end{tabular}

B2 Morin (2011)

Serving as magistrate at the French Cour des comptes: Navigating between tradition and modernity

B3 Mutiganda (2013)

Budgetary governance

B4 Caramanis et al. Transplanting Anglo(2015) American accounting oversight boards to a diverse institutional

B5 Contrafatto context (2014) public sector organizations: An institutional and critical realism approach

AAAJ Canada

Journal Location Findings

$A A A J$ Finland

"Both the interview transcripts and all the published material are in Finnish and hence the quotations provided in this paper have been translated by the authors."

(p. 1115)

"We try to safeguard public financial resources, to keep the State from overheating, to limit the administrative greenhouse effect, to indicate new ways of saving the energy of State agents ...' (our translation)." (p. 728)

CPA Finland "[T]he author translated all quotes not made in English." (p. 523)

"Interviewees expressed themselves either in Finnish, Swedish or English, depending on their choice. Interview summaries, however, were written in English." (p. 524)

AOS Greece/UK "In addition, all semi-structured interviews cited herein have been conducted by the other coauthors." (p. 17)

Italy "Interviews were carried out, social and environmental reporting: An Italian narrative

B6 Brivot and Beyond panopticism: On AOS Canada Gendron (2011) the ramifications of surveillance in a contemporary professional setting

B7 Skærbæk and The role of accounting Tryggestad (2010) devices in performing through the medium of the Italian language ...." (p. 419) "Quotations from interviewees and other evidential sources, which are reported in the following narrative, have been translated from Italian to English by the author." (p. 419) "All interviews were in French; we translated the excerpts incorporated in this paper." (p. 143)

\section{Table II.}

Findings dealing corporate strategy

B8 Hansson and Contracting Longva (2014) accountability in network governance structures

QRAM Sweden/ Norway
"All quotations from the case account are translated from Danish by the authors." (p. 112) "The working groups were there before, but many of them had not been functioning due to lack of money. Now they are back in operation because of the money supply - there is a big difference! (Interview I, translated).” (p. 104) 


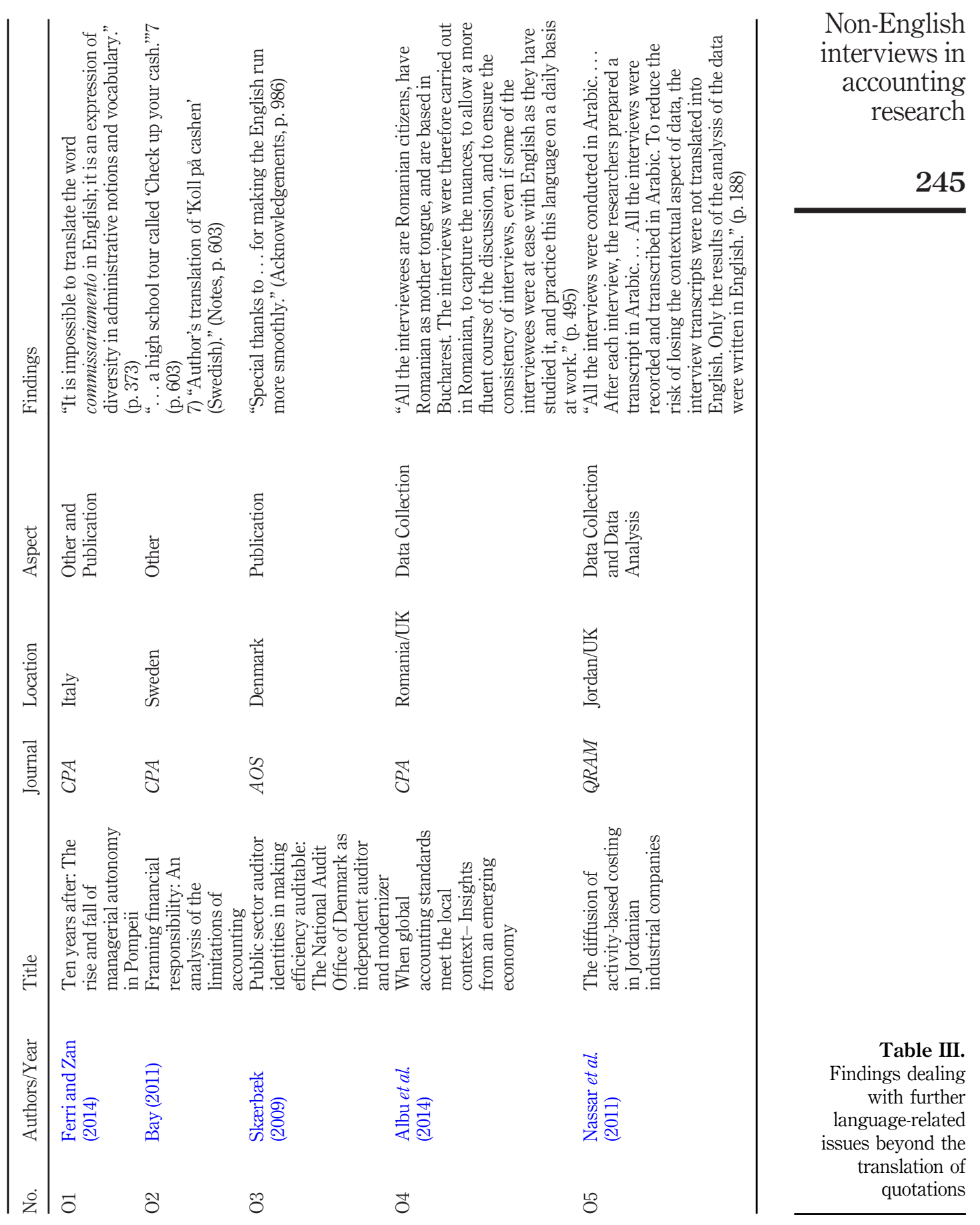


QRAM

17,2

\section{6}

translation aspects during data collection was given in the methods section of the corresponding paper. Other aspects on translation and translation issues can be found throughout the paper (e.g. in the notes or acknowledgements, or directly following the interview quotations).

Our main findings of the literature analysis can be summarized as follows:

(1) In 17 per cent (41 out of 246) of the analyzed papers, notes on translation in some form can be found. In turn, in 83 per cent of cases (205 out of 246), absolutely no information is presented on the language in which the interviews were carried out or how they were translated; and

(2) In only 3 per cent ( 8 out of 246) of the analyzed papers do the authors comment on the actual translation of quotations from non-English interview data in the publication. In these cases, for example, the language in which the interviews were conducted was noted along with who carried out the translation.

\section{Interviews with established researchers}

Our interview findings are in line with the findings from our literature analysis, but they reveal some additional insights into the motivations for (not) reporting on translating quotations in interview-based accounting studies. Similar to the 3 per cent (8 out of 246) analyzed papers in which the language of the interviews and the person responsible for the translation are mentioned, we asked our interviewees in which countries and in which languages their interviews (in the studies included in our sample) were conducted. All of our seven interviewees (Interviewees 1 to 7 ) stated that the interviews were conducted in the country of their primary affiliation, and therefore in the official local language of their affiliation, which confirms $A 1$. For instance, one of our interviewees had conducted interviews in Germany in German, another in Romania in Romanian, and a third in Finland in Finish. All interviewees explained that they themselves had translated the provided quotations and interview passages. Only one of our interviewees (Interviewee 6) noted that the direct quotations were then sent to a professional language/translation service in the source and the target language, to check the quality of the translation.

Based on our literature analysis, we could not identify reasons why only a small minority of papers reported on language and translation issues. In the interviews, we aimed to shed some light on this issue and explore whether potential translation issues were ignored or just not reported. The collective evidence from our interviews suggests that although the reporting on translation issues is not (yet) widespread, some researchers' awareness of these issues seems to be growing.

Our interviewees explained why they have not yet report on translation issues. Interviewee 1 explained that he/she did not view such issues as being important in the paper included in our sample ("I don't think there was an effect"), whereas most other interviewees mentioned that their awareness of such issues had been low so far, noting, for instance, "I have not done it [.. . ] there is no particular reason for that, I mean, sometimes [. . .] I forgot writing that or I didn't feel it important to write" (Interviewee 2), "we didn't think it would be an issue" (Interviewee 3), or "we haven't reflected on that" (Interviewee 6). Others pointed to prevailing informal standards in reporting on methods of interview-based accounting studies. Interviewee 3, for example, said that when writing up a methods section of the paper, he was much oriented towards "the mainstream [...]. And in our field, in management accounting, those studies typically never address translation issues." Similarly, Interviewee 2 noted that accounting researchers do not normally report in detail on methodological issues, which are assumed to be irrelevant. In his view, this also applied 
to the translation of interview quotes in his study, which is part of our literature analysis sample.

At the same time, some of our interviewees reported that they had critically reflected on the use of language, translations and their reporting on these topics in publications. For example, Interviewee 1 has subsequently changed his reporting behavior on language and translation-related aspects in his studies. He explained this change in behavior as the result of a "discussion in our [.. . t team when we were translating [. . . one of the quotations." Also, Interviewees 6 and 7 said that in retrospect, they should have reported on language and translation of interview quotations in more depth, for instance by explicitly naming the language of data collection and who translated the data for analysis and publication. As argued by our interviewees, reporting on such issues can increase the transparency (Interviewees 1, 6, 7), plausibility (Interviewee 4), rigor (Interviewee 3), and reliability (Interviewee 4) of the underlying research. Put differently, according to Interviewee 1, not reporting on language and translation issues could create some kind of "distrustworthiness" of the research paper due to a lack of evaluation regarding whether the reconstruction of meaning has been achieved - the latter being a key quality criterion for the translation of quotations, as explained above.

Therefore, in general, there is some agreement among our interviewees that a transparent reporting on the translation in general and in particular, of quotations would be an important ingredient in qualitative, interview-based accounting research projects. Interviewee 4 even went further and suggested that reporting on language and translation issues is an essential part of any kind of research and thus not limited to qualitative research or to specific research contexts[13]. While this aspiration is certainly beyond our research focus here, Interviewee 1 suggested that the reporting on language and translation issues in qualitative research should at least be treated like a "control variable" in quantitative research, one "that shows, that you have a bias-free translation and that is important".

Besides the overall importance of such reporting, the context-sensitivity of interdisciplinary accounting research has also been noted by our interviewees. That is, Interviewees 3, 6, and 7 argued that especially in situations of significant differences between the source culture/language and the target culture/language, information on language and translations should be reported to some orientation and pre-understanding of the reader towards the research context and the provided quotations. For instance, Interviewee 6 stated that translation from one culture to another could be difficult because the information given by interviewees is "contextualized." In line with this notion, Interviewee 2 said that reporting on the "institutional background [...] of the people" interviewed would improve reader understanding.

To illustrate the consequences of higher or lower perceived context-sensitivity, consider the case of two of our seven interviewees, both of whom conducted interviews with civil servants. Together with his co-authors, one interviewee had conducted interviews with "Beamte" in Germany. In the paper included in our literature analysis sample, it is explained in brackets that "Beamte" is the German term for "civil servant," but the rest of the paper refers to this group as "Beamte" and the term remains untranslated. In our conversation with the interviewee, he mentioned that the reason for this approach was that they regarded the term "Beamte" as "untranslatable" and specific to the German context. Their study was also very much focused on the identity reconfiguration of these "Beamte", which - according to the interviewee - further necessitated keeping the original German term. In contrast, another interviewee, together with her co-author, conducted interviews with Swedish and Norwegian "civil servants," and decided to use this term without reporting the equivalent Swedish or Norwegian ones. In this second study, however, the civil servants' identity 
QRAM 17,2

played a less prominent role than in the first. What these two examples show is that both of our interviewees were confronted with the decision whether or not to translate the German, Swedish, and Norwegian equivalents for "civil servants," and decided to handle this problem in different ways. We cannot judge whether the context in the first study was so specific as to require the non-translation of "Beamte", and the second study's context was rather unspecific regarding the term "civil servant", but what this example serves to indicate is that different accounting researchers may interpret context-sensitivity differently. A further difference between the two cases is that the authors of the second study had relied on the help of a professional translator. That is, our interviewee mentioned that she had sent the self-translated manuscript and the original quotes in Swedish to the professional translator to check it for proper English and a proper translation of the quotations. In contrast, the other interviewee had not let the translation of the interview text be checked by a professional translator. This does not necessarily mean that due to the involvement of the professional translator, the translation of the respective source-language equivalents for "civil servants" would have played out differently, but in our view, this comparative example shows that for translations in accounting research, the person who carries out or checks the translation may impact differently the outcome of translations.

With reference to direct quotations, however, not only the context seems to be relevant, but also what was said and how. For instance, Interviewee 2 argued that:

$[\ldots]$ not only the $[\ldots]$ content of the quote is relevant but also [...] how the original quote was phrased, [...] the phrasing, the words and the entire intonation of the quotation is relevant for interpreting it.

When translating direct quotes for her publication, for Interviewee 6 it was important first "to keep the nuances in the interviews" and second to "capture the nuances in the quotations." Such considerations were also highlighted by Interviewee 1, who stated that:

[...] our focus is on translating so that the meaning is exactly the same in English that it was in German. That does not mean that we change completely a sentence or a word or so, but sometimes, if you have alternatives to translate one word, we try to choose the word that transports the meaning of the sentence.

Interviewee 2 described his procedures similarly: "I tried to translate in a way that [...] it adjusted to the original quote as much as possible".

The above interviewee statements suggest that no single "right" translation exists for quotations that can undoubtedly transfer the context, meaning and phrasing. In line with previous translation studies and the methodological accounting literature (Harkness, 2003), our interviews therefore confirm that translation in terms of the transfer of meaning remains an act of sense-making, reconstruction and interpretation. Further, our interviewees also seem to subscribe to the notion that translation is about finding a translation that creates "equivalent effects on the target reader" (Evans et al., 2015, p. 28; Nida, 1993). This understanding implies that different translation alternatives can achieve this aim. Indeed, different translation approaches can tackle these issues, as mentioned by Interviewee 1:

We have different [...] options for how to translate [the different psychological concepts of pressure]. Of course, the most obvious one is a literal translation, but that does not make any sense.

For Interviewee 1, a literal translation would aim for equivalence in terms of lexis. Instead of a literal translation in terms of lexical equivalence, which did not make any sense for these authors, Interviewee 1 and his co-authors decided to translate the psychological concepts of pressure using a more functionalist approach. Interviewee 1 noted that they "try to [...] 
transport the meaning of the sentence" and "the background of our contents, the context where this quote was delivered, so that it makes sense and transfers the [.] main points." As indicated above, such close consideration of the translation of interview quotes was new to Interviewee 1 in the specific research project involving psychological concepts of pressure. From intensive discussions with his co-authors, Interviewee 1 noted that he became aware of the challenges of translating interview quotations and changed his reporting behavior in his subsequent studies.

In trying to open the "black box" of translation processes and strategies in qualitative interview-based accounting research, we also asked our interviewees to describe explicitly their translation processes when publishing their results (in general and for quotations). Surprisingly, all interviewees answered this question on process by mentioning the person of the translator first. Hence, our interviews suggest that the person of the translator plays a crucial role in the translation process. While the person who carried out the translation cannot be equated with the whole translation process, this evidence suggests that the person of the translator is a relevant issue in qualitative interview-based accounting research. For this reason, we return to this aspect when developing our recommendations.

In connection with the person responsible for the translation, the importance of the researcher as a translator and his/her language and translation skills and experience were stressed by one interviewee. Interviewee 7 mentioned that she had studied English as a major at university and had attained professional skills in English. In her paper, she mentioned that she had self-translated the quotations, but did not report her professional English-language skills. Subsequently, in our interview with her, she acknowledged that it would have been important for the reader to know that she had "almost professional knowledge of English" and thus "was able to carry out the translation by [her]self." Owing to her skill in the target language, she subsequently argued that:

[...] it might have been important to inform the reader that one of the authors had almost professional knowledge of English because that, of course, makes a difference [for the result of the translation].

Therefore, in line with the abovementioned relevance of the person who translated the quotations, Interviewee 7 argued that information on the translator could "help the reader have an orientation toward the quotes".

\section{Discussion and recommendations}

Discussion of findings

Our findings suggest that the vast majority of interdisciplinary accounting studies relying on interviews not conducted in the English language do not comment on translation issues in general. Our findings further indicate that this is also true for the more specific case of reporting on the translation of quotations from interviews. To summarize, our findings from the literature analysis and the interviews support Steyaert and Janssens (2012) critique of the unreflective usage of language in general - and the English language in particular - in business-related academic research (Evans et al., 2015). Furthermore, our findings concur with the recent review by Aguinis and Solarino (2019), who criticize the lack of transparency in interview-based strategic management research and argue that this lack of transparency negatively affects the trustworthiness and replicability of such studies.

Only a small proportion of the papers covered in our literature analysis contain information on translation-related issues, and an even smaller proportion disclose the language of data collection and information on who translated the quotations from interviews. In turn, most of the papers containing some indications of language issues seem
Non-English interviews in accounting research 


\section{QRAM} 17,2 to have paid attention to translation issues in an early stage of the research process. For instance, Albu et al. (2014, p. 495; O4 in the above table) disclose the language of data collection: "all the interviewees are Romanian citizens, have Romanian as mother tongue [...]. The interviews were therefore carried out in Romanian, to capture the nuances." This statement suggests that the language of data collection can be an important factor to capture possible nuances of social knowledge and reality expressed in interviews. Similar to Albu et al. (2014), other authors in our literature analysis sample disclosed the language of data collection and thus a language-related issue situated in the earlier parts of the research process.

Our very limited findings in the literature analysis on language issues in further steps of the research process may indicate that the awareness regarding language-related issues becomes lower in later stages of the research process (Marschan-Piekkari and Reis, 2009). However, data analysis and the publication of results are also sensitive to language and translation issues. For instance, Nassar et al. (2011, p. 188; O5 above) explained that:

[...] [a]11 the interviews were conducted in Arabic. [...] After each interview, the researchers prepared a transcript in Arabic. [...] All the interviews were recorded and transcribed in Arabic. To reduce the risk of losing the contextual aspect of data, the interview transcripts were not translated into English. Only the results of the analysis of the data were written in English.

Therefore, in this case, the authors tried to preserve the cultural context of their interview data by keeping data analysis in the source language. Given that authenticity, a key quality criterion for qualitative accounting studies, can be achieved by preserving and communicating the richness of collected interview data (Messner et al., 2017), such reporting on language procedures as exhibited in the study by Nassar et al. (2011) can support the authenticity of qualitative, interview-based accounting research.

These few examples indicate that there is at least some sensibility toward and awareness of the relevance of the translation and corresponding reporting issues. This increasing awareness of the role and importance of language and translation-related issues - although potentially still in its infancy - is also reflected in our interviews. However, our literature analysis and interviews reveal that language sensitivity seems to get lost somewhere when it comes to the final step of the research process, the publication of results. In particular, we could only identify very limited information (in 3 per cent of the papers in our literature analysis) on the treatment of quotations from interviews in final papers. This observation is in line with Dai et al. (2019, p. 6), who found that:

[...] [i]t is an irony that in contrast to the deep analytical and rich description skills often associated with qualitative research [.. . ] a non-trivial number of qualitative researchers [...] were lacing on applying these abilities in their presentations of their methods.

Our interviews indicate that this observation is also true for reporting on the translation of interview quotations, although our interviewees have mentioned numerous advantages that are associated with reporting on language and translation issues.

Given these advantages, along with the fact that we purposefully analyzed four interdisciplinary accounting journals, the limited findings on the disclosure of translation processes in our literature can be considered somewhat surprising. Highly cited articles in these journals have frequently advocated a view that is sensitive to the context in which accounting operates and in which accounting phenomena can be observed (Burchell et al., 1985; Flamholtz, 1983; Hopwood, 1983; Laughlin, 1988; Ramirez, 2001). In general, the context specificity of the research findings is often stressed in interdisciplinary accounting research (Burns, 2000; Burns and Baldvinsdottir, 2005; Hiebl, 2018; Hopper et al., 2009). As explained above, to take effective care of such a context when transferring interview-based 
findings from one culture to another, translation requires specific attention. Thus, to transport the meaning of context-specific quotations from the source language to the target language, a closer consideration of and reporting on translation procedures may be warranted - which was also reflected in some of our interviews with senior accounting scholars.

On a more general note, our findings support and extend those of Evans et al. (2015), who suggest that the consideration of translation in accounting practice is scant. We add to this finding by showing that the consideration of language and translation issues may also be limited in qualitative interdisciplinary accounting research. This seems regrettable, since in both research and practice, deficient translations may be harmful (Evans, 2016; Evans et al., 2015; Kettunen, 2017). Most importantly, such deficient translations - including the neglect of issues pertaining to the cultural context - may significantly impact the results reported in qualitative accounting studies. Given the relevance of translations and the limited consideration so far, in the following section we develop recommendations for reporting on the translation of quotations from interviews for future accounting research based on interviews conducted in languages other than English.

\section{Recommendations for future accounting research}

A quality criterion for translating interview data for publications in accounting research is the reconstruction of "powerful" quotations that can:

- ensure the survival of the original meaning and sense of what has been said in the source language; and

- sufficiently consider the context-sensitivity of such quotes.

As translation studies suggest, the translation process is characterized by sense making and the re-expression of ideas. Therefore, multi-modal reconstruction and interpretation (Salama-Carr, 2009; Simon, 1997) should lead to a translation that creates equivalent effects for the Englishlanguage reader to those for the source-language reader (Evans et al., 2015; Nida, 1993; Xian, 2008). While we refer to translation as an act of multi-modal reconstruction, interpretation and sense making, the notion of the "transfer of meaning" should be understood as capturing the abovementioned aspects. This understanding of the "transfer of meaning" and its complexity also means that there is no single "correct" translation; different ways to achieve a "transfer of meaning" exist. Hence, the translation of quotes depends on the translator finding a translation with which he/she is "comfortable" (Pentland, 1993). The potential loss of meaning during translation was also mentioned as a relevant issue by most of our interviewees, especially the survival of captured, specific nuances, which were stressed in this context. The necessary multimodal reconstruction of meaning, sense, context and ideas seems particularly important in qualitative studies to increase the authenticity of the data (Messner et al., 2017). Moreover, and as explained by Xian (2008, p. 236), "the translated data should produce a response in the reader in a target language of publication that is essentially like the response to the reader in the original culture." However, as noted by Shapiro et al. (2007, p. 261), "[t]he reality is that academic journals that act primarily as knowledge sources are uncertain about how knowledge in published articles will be received, interpreted and used." Readers of such articles may also be unable to evaluate whether the "transfer of meaning" as a multi-modal reconstruction has actually been achieved through a context-sensitive quotation that reconstructs the subtle terms and nuances expressed by interviewees. Such inability may produce the perception of lower credibility and authenticity of the underlying data and therefore the underlying research: first, the readers usually do not know what interviewees wanted to convey in their entire interviews or in their individual quotations, because based on the readers' own language and translation skills, they might be 
QRAM 17,2

unable to assess the quality of a translation in terms of "the transfer of meaning". Second, as translation studies suggest, a translation into the target language is only a close approximation of the text in the source language (Evans et al., 2015; Xian, 2008). Considering translation as a multimodal act that constructs meaning, sense, ideas and context using subtle terms and nuances, multiple translations might fit these aspects. Hence, it seems unlikely that any translation is the only true and correct translation, which further necessitates the disclosure of the taken translation approach.

As the broader audience might be unable to assess the quality of translations in terms of a "transfer of meaning", other criteria may be taken into consideration to allow readers to draw their own conclusions on the quality of the translation process (Santos et al., 2015; Temple and Young, 2004; Pugh and Vetere, 2009). In the following, we focus on the transparency of the underlying translation process with specific regard to the translation of interview data for direct quotation in publications (Enzenhofer and Resch, 2011). In line with translation studies, we therefore assume that the reporting on the translation process, especially with regard to the translation of direct interview quotations, can give an indication of the quality of translation and of the researchers' awareness of and sensibility toward translation issues.

Transparency can be achieved by disclosing and reporting on the translation treatment in the method sections of publications. Such transparency is a valuable opportunity to establish the credibility and authenticity of a qualitative interview-based study (Messner et al., 2017). In line with this notion, Dai et al. (2019, p. 11) argue that "transparency is frequently presented as an inevitable passage point to ensure trustworthiness or "rigor" of qualitative research." While the transparency of translation processes may not enable readers to assess the actual quality of the translation, they might at least be informed of the efforts to ensure quality translations. Readers might also be able to assess whether the authors met the key requirements or scientific standards for translations. If language, translation issues, and the translation process were made transparent, readers could at least infer whether the authors have taken sufficient care to enable a successful multi-modal reconstruction of what the interviewees said, considering the transfer of meaning, sense and context. In addition, the requirement to make translation processes transparent could also strengthen researchers' awareness of and sensibility for language sensitive contexts and translation problems while preparing data for publication.

In this context, we make the following recommendation on reporting for future accounting research by non-native English researchers when translating quotations from non-English interviews:

All relevant information on the translation process of interview quotations and
related issues should be made transparent in a manuscript's methods section.
Such information should at least include: (a) the chosen translation approach and related issues, such as information on how the translation process sought to take into account the context in which meaning was expressed, and.

(b) the languages in which the data collection and data analyses were carried out. A further piece of information that could be disclosed in the methods section, but that cannot be seen to be as important as (a) and (b) and that should thus be seen as optional:

(c) the person (and his/her translation experience and skills) or the translation agency that carried out the translation. 
Reporting on such relevant information allows the readers to make sense of the quality of the translation process and the level of detail applied, and - given the importance of quotations for interview-based studies (Messner et al., 2017; Scapens, 2004) - it should strengthen a study's overall credibility and authenticity. Furthermore, we do not see transparency as an end in itself. Our reporting recommendation should be understood as an opportunity to raise researchers' awareness of and sensibility toward the language- and translation-related issues that could arise in the research process in general as well as when preparing quotations for publication.

Reporting on the chosen translation approach and related issues, as suggested in (a), should be of interest to the readers since it makes transparent how translation decisions have been made. This recommendation subsumes the disclosure of the way in which translation challenges were addressed, how the pitfalls of the translation process have been taken into consideration and avoided or reduced, if there are other uncertainties surrounding the translation, and any other efforts undertaken to increase the quality of the translation process, especially with regard to the translation of quotations. Reporting on such issues could also include the handling of words with different nuances. This notion does not necessarily mean there is only one true and correct translation. Owing to the person of the translator and chosen translation approach, as explained in this recommendation (a) and in the optional recommendation (c), different translation alternatives might be suitable in a given context. Another example of how to deal with those translation issues and processes regarding quotations has been addressed by Interviewee 1, whose study included different "psychological concepts of pressure". In consequence, as explained by Interviewee 1, related translation problems for the direct quotations in the publication led to internal discussions in the author team and resulted in a changed reporting behavior on language and translation issues in his subsequent publications.

We would envisage that information in line with (a) also includes the handling of translation issues in cases where translation of the interview data might have proven difficult. For instance, difficulties can arise because there are no equivalent words in the source and the target language, and a description of what has been said in an indirect quotation might lead to a loss of connotation, nuances, and meaning. Because some words do not have close equivalents in different languages and cultures (Simon, 1997; Holland and Ramazanoglu, 1994), some interview passages might not be translated, but be kept in the source language (Xian, 2008) - recall "Beamte" in the study discussed above. Similar issues that arise in the translation of interview quotations should not be simply dismissed, but instead should be recognized and mentioned (Enzenhofer and Resch, 2011; Steyaert and Janssens, 2012), and even treated as an essential piece of information that could reveal differences in worldviews. It might be precisely this kind of information that the researchers wanted to address by conducting interviews, which allow them to capture not only facts but also emotions, attitudes, and subtle notions. For instance, among the papers we analyzed, Ferri and Zan (2014, p. 373) noted that "[i]t is impossible to translate the word commissariamento in English, as it is an expression of diversity in administrative notions and vocabulary".

In (b), we suggest disclosing the languages used at different stages of the research process. As noted by González y González and Lincoln (2006, p. 2):

$[\ldots]$ the collection of data in a local language, and the presentation of the analysis in a second language, becomes an important issue to consider. The presentation of data analyses and findings is a huge undertaking for any researcher who hopes to make certain that readers understand and make sense of data.
Non-English interviews in accounting research

253 
QRAM 17,2

In line with this notion, translations can be regarded as a potential source of bias in the research process. With regard to the different research stages, at every interface between them (data collection, data analysis, publication of results), translation issues might occur. If the language of data collection differs from the language of data analysis, which in turn differs from the publication language, multi-translation loops complicate the translation of interview quotations. In such situations, translation has to be performed twice: from the language of data collection to the language of data analysis, and afterwards to the language of publication. As a consequence, the risk of translation errors and thus bias rises, and the "richness of quotations" might not survive multiple translation loops (Mcdonald and Hellgren, 2009, p. 272). Such multi-translation loops differ from translations of interview quotations, which only consider the source language and thus not the language of data analysis. The latter might be the case if interview transcripts were written in a language other than the one in which the interviews were conducted. As far as can be ascertained from the examined articles and our interviews, single-translation loops are mostly present in qualitative, interdisciplinary accounting research. Although without increased transparency, readers are unable to assess if the "transfer of meaning" throughout the translations has been achieved, and so making the amount of translation loops and correlated translation issues transparent could enable the readers to draw conclusions on the risks involved in translations. Therefore, the language of data collection, the language of data analysis, and the number of translation loops should be disclosed. For instance, such disclosure in line with (b) can be observed in the study by Mutiganda (2013; A3 above), who explains that interviewees were allowed to express themselves in Swedish, Finnish, or English, while the interview summaries and the publication were written in English.

As recommended in (c) as optional information, disclosing the person (and his/her translation experience and language skills) or agency responsible for the translation may be an important piece of information. Returning to our earlier civil-servant example and the case of Interviewee 7 who had studied English as her major, the person who carries out the translation may influence the outcome of the translation considerably. The importance of the person who translates the quotations was also addressed by all remaining interviewees. All of them started to answer the question about how the translation process was organized by mentioning the person responsible for the final translation. While the translator should not be equated with the translation process per se, the notion that he/she plays an important role in this process concurs with the findings of translation studies (Temple, 2002, 2008; Temple and Young, 2004; Xian, 2008). This literature suggests that the person carrying out the translation conveys a message from the source to the target language while reconstructing the meaning of what has been said. The personal background of the translator thus influences the outcome of the translation, which results in a notion of meaning that is more dynamic and fluid than the rather technical view that one true and correct translation can be achieved.

Further underpinning the relevance of the translating person, Enzenhofer and Resch (2011) argue that professional translators may differ very much from lay translators. For example, compared with lay translators, professional translators may differ in their knowledge of translation approaches and the implications arising from following one approach or another. Consequently, the decision on which translation strategy [see (a)] is suitable for achieving a proper "transfer of meaning" - just as further translation decisions and challenges - may differ between lay and professional translators. We do not argue that the employment of a professional translator is the ideal practice for providing a translation of meaning with which the researcher is comfortable. A suitable transfer of meaning can most likely be achieved if the person has professional competencies in the relevant research 
domain (accounting, in this case), language skills (source language and English) and context-sensitivity. This can be achieved either by a professional translator or the researcher or by a strong cooperation between the two, which was also mentioned by one of our interviewees (Interviewee 6). Given this potential importance of the person(s) conducting the translation (and their language skills and translation experience), the person or agency having carried out the translation could be made transparent in the methods section, as per recommendation (c). In the case of the researcher as a translator, such information could include statements on the source- and target-language skills (see the case of Interviewee 7

Non-English interviews in accounting research who has professional English-language skills from having studied English as a major at university) or years of experience in working in the source culture (Xian, 2008). In the case of a hired professional translator, such information could include the fact that an agency has been consulted and why that agency has been chosen (Santos et al., 2015)[14].

\section{Conclusions}

The purpose of this paper was to examine the methodological consideration of the translation of non-English interview quotations for publication in interdisciplinary Englishlanguage accounting journals. From our review of related literature published in four such journals, we conclude that translation is more than just finding the right words; rather, it is a complex task of transferring meaning and sense as well as the contextual setting of the interview and interviewee. This is of specific relevance for translating non-English interview quotations, which are, for instance, used to highlight findings through the presentation of the subtleties and notions of interviewees.

Our analysis of 246 articles in four interdisciplinary accounting journals ( $A A A J, A O S$, $C P A$ and $Q R A M)$ indicates that translation issues regarding interview data were made transparent in only a small minority of the analyzed publications. Few works explicitly refer to translation issues and even fewer to the translation of interview quotations. It can therefore be assumed that the nature of language and translation is an underestimated topic in qualitative, interdisciplinary accounting research, a notion that was also reinforced by our interviews with experienced accounting scholars. The low awareness of translation issues seems surprising since interdisciplinary accounting research usually emphasizes the context specificity of the research findings (Burns, 2000; Flamholtz, 1983; Hiebl, 2018; Hopper et al., 2009) more than, say, more positivist accounting research. In addition, as expressed by Harkness (2003, p. 56), "interdisciplinary input is long overdue; developments in linguistics, translation studies, and pragmatics have much to offer." This is why we conclude from our analyses that greater consideration of translation issues in interdisciplinary accounting research is needed.

In combining developments from translation studies and accounting research, this paper's main contribution to the accounting literature are some concrete recommendations for future accounting research dealing with reporting translations of non-English interview quotations. These recommendations share the goal of:

- raising the awareness of and sensibility toward language- and translation-related issues in the research process; and

- increasing transparency when reporting interview quotations in interdisciplinary accounting research.

For the case of the translation of interview quotations, our recommendations therefore specify how the transparency of qualitative, interview-based accounting research may be increased and thereby go beyond more general advice on increasing transparency in interview-based research offered by prior research (Dai et al., 2019; Aguinis and Solarino, 
QRAM 17,2

2019). Potentially, as envisioned by Evans and Kamla (2018) and Aguinis and Solarino (2019), journal editors may also find some inspiration in our recommendations when setting up or developing journal policies regarding translation issues, as most journals do not (yet) have policies on language and translation (Steyaert and Janssens, 2012).

In turn, we assume that by following our recommendations, a study will benefit in terms of overall credibility and authenticity, which are key quality criteria in interview-based accounting research (Messner et al., 2017). In addition, following our recommendations should also enable readers of the final publications to assess better the quality of the translations they encounter. It is hoped that more non-native English researchers aiming to publish their research results in English-language accounting journals follow these recommendations in the future, as this would likely lead to research methods being more transparent and may contribute to reducing biases that are the result of translation issues. Thus, such measures would stand a good chance of enhancing the scientific quality of accounting papers based on non-English interviews.

\section{Notes}

1. We must note at this early stage that it is not within the scope of this paper to examine whether sufficient English-language skills and competencies are a success factor for getting published in highly ranked (English-language) journals.

2. We acknowledge that translations of interviews for direct quotations might not be the only interface in the research process that could be problematic for translation. For interviews not conducted in English language, language and translation issues might be especially relevant, as the quotations are commonly provided in English.

3. Another important aspect of qualitative accounting research is "reflexivity." Because of word count restrictions, we will not detail this concept here, but we refer the reader to Parker (2012).

4. Beside those translation strategies, other exemplarily translation related problems could be found in the following sections. With reference to translation related problems at different stages of the research process, see Temple (2008), Steyaert and Janssens (2012), Welch and Piekkari (2006) and Xian (2008).

5. We acknowledge that the transfer of meaning is not the ideal intended goal of all translations. For instance, focusing on the transfer of meaning may not apply to the translation of poems in which verse form and rhythm must be considered besides content. However, with regard to the translation of quotations and based on methodological considerations, translation studies, and linguistics, we can assume that the transfer of meaning might be an adequate approach for most interview-based studies in interdisciplinary accounting research.

6. For an overview of other examples dealing with language and translation related issues in other disciplines, which could be relevant for accounting research, see Evans et al. (2015).

7. We acknowledge that other journals such as European Accounting Review and Management Accounting Research are open to qualitative studies, too (Dai et al., 2019).

8. In our preliminary analyses, we checked for the erroneous exclusion of articles from our study due to assumption $A 1$. We used articles published in 2015 for this preliminary analysis. We identified 22 papers published in 2015 in the four selected journals, which were written by authors located in a country whose official language is English. Following A1, these papers were excluded from our sample, as no translation issues would arise. The 22 papers were screened to identify the language in which the interviews were conducted and subjected to an in-depth analysis. We could only identify three of these 22 papers (i.e., 14 per cent) in which the language of the interviewees did not clearly match the official language of the authors' affiliation (English in this case). This means that in 2015, only three papers of 22 were erroneously excluded from our sample due to A1. Hence, the result of our preliminary analysis was that only in 14 per cent of 
the cases from 2015 did A1 not hold, which is, in our view, a sufficiently low share to warrant the retention of A1 in our full literature analysis.

9. Of course, there remains the possibility that, for instance, interviews were conducted in Italy, but in the English language. As reported in the methods section, our findings reveal that in the timeframe we analysed, such cases seldom occurred in the interdisciplinary accounting literature. That is, there were only 20 papers which we excluded from further analysis due to relying on English-language interviews where no translation issues arose.

10. For AAAJ via EmeraldInsight, www.emeraldinsight.com/loi/aaaj; for AOS via ScienceDirect, www. sciencedirect.com/science/journal/03613682; for CPA via ScienceDirect, www.sciencedirect.com/ science/journal/10452354; for QRAM via EmeraldInsight, www.emeraldinsight.com/loi/qram.

11. Not only were the sections entitled "method" read, but also their equivalents (i.e., research design, methodology, design of study, research approach).

12. In total, we asked 15 experienced researchers in interdisciplinary accounting research, whose papers are part of our literature analysis and whose mother tongue is not English, to participate in our interviews. Seven of them agreed to take part in an interview with us.

13. Following Interviewee 4, reporting on language and translation issues is an essential part of any kind of research, and should not be limited to qualitative research. Although beyond the scope of our investigation here, insights from translation studies and linguistics suggest similar things. That is, there are calls that language and translation issues and their reporting are essential parts for any kind of research. This should not be limited to specific research settings or other contexts, even those that might be especially relevant. At least, Evans and Kamla's (2018) and Steyaert and Janssens (2012) calls for journal policies on such issues should highlight the importance of language and translation aspects in general, independent of the research setting, topic, etc.

14. We acknowledge that "hidden translation work" might exist (and we thank one of the reviewers for providing us with this insight). In such work, translated quotes in the target language might be revised and modified by, for instance, copy editors during the proofreading process to make the English flow more smoothly. Our own experience of translating interview quotations for publication shows that copy editors sometimes make suggestions to improve interview quotations that have already been translated into English by the authors - without the copy editor knowing the sourcelanguage quote. Such edits may also influence and change the meaning purveyed in a quotation without having engaged a translation agency. If, however, fellow accounting researchers followed recommendations $(a)$ and $(c)$, such hidden translation work would also be made transparent when reporting how the translation process was organized and who participated in it.

\section{References}

Aguinis, H. and Solarino, A.M. (2019), "Transparency and replicability in qualitative research: the case of interviews with elite informants", Strategic Management Journal, Vol. 40 No. 8, pp. 1291-1315.

Albu, C.N., Albu, N. and Alexander, D. (2014), "When global accounting standards meet the local context - insights from an emerging economy", Critical Perspectives on Accounting, Vol. 25 No. 6, pp. 489-510.

Alexander, D. (1993), “A European true and fair view?”, European Accounting Review, Vol. 5 No. 3, pp. 483-493.

Bagranoff, N.A., Houghton, K.A. and Hronsky, J. (1994), "The structure and meaning in accounting: a cross-cultural experiment", Behavioral Research in Accounting, Vol. 6 Supplement, pp. 35-57.

Baskerville, R. and Evans, L. (2011), The Darkening Glass: Issues for Translation of IFRS, Institute of Chartered Accountants of Scotland, Edinburgh.

Bay, C. (2011), "Framing financial responsibility: an analysis of the limitations of accounting", Critical Perspectives on Accounting, Vol. 22 No. 6, pp. 593-607.

Non-English interviews in accounting research 
QRAM 17,2

Blackwell, N.L., Perlman, M. and Tree, J.E.F. (2015), “Quotation as a multimodal construction”, Journal of Pragmatics, Vol. 81, pp. 1-7.

Blenkinsopp, J. and Pajouh, M.S. (2010), "Lost in translation? Culture, language and the role of the translator in international business", Critical Perspectives on International Business, Vol. 6 No. 1, pp. 38-52.

Brislin, R.W. (1970), "Back-translation for cross-cultural research”, Journal of Cross-Cultural Psychology, Vol. 1 No. 3, pp. 185-216.

Brivot, M. and Gendron, Y. (2011), "Beyond panopticism: on the ramifications of surveillance in a contemporary professional setting”, Accounting, Organizations and Society, Vol. 36 No. 3, pp. 135-155.

Burchell, S., Clubb, C. and Hopwood, A.G. (1985), "Accounting in its social context: towards a history of value added in the United Kingdom”, Accounting, Organizations and Society, Vol. 10 No. 4, pp. 381-413.

Burns, J. (2000), "The dynamics of accounting change inter-play between new practices, routines, institutions, power and politics", Accounting, Auditing and Accountability Journal, Vol. 13 No. 5, pp. 566-596.

Burns, J. and Baldvinsdottir, G. (2005), “An institutional perspective of accountants' new roles: the interplay of contradictions and praxis", European Accounting Review, Vol. 14 No. 4, pp. 725-757.

Caramanis, C., Dedoulis, D. and Leventis, S. (2015), "Transplanting Anglo-American accounting oversight boards to a diverse institutional context", Accounting, Organizations and Society, Vol. 42, pp. 12-31.

Condie, J. (2012), "Beyond rationalisations: improving interview data quality", Qualitative Research in Accounting and Management, Vol. 9 No. 2, pp. 168-193.

Contrafatto, M. (2014), "The institutionalization of social and environmental reporting: an Italian narrative", Accounting, Organizations and Society, Vol. 39 No. 6, pp. 414-432.

Corbeil, J.P. and Blaser, C. (2007), The Evolving Linguistic Portrait, 2006 Census, Statistics Canada, Ottawa.

Covaleski, M.A., Dirsmith, M.W. and Samuel, S. (2017), "Analysing and interpreting qualitative data in management accounting research”, in Hoque, Z., Parker, L.D., Covaleski, M.A. and Haynes, K. (Eds), The Routledge Companion to Qualitative Accounting Research Methods, Routledge, New York, NY, pp. 387-404.

Dai, T.D., Free, C. and Gendron, Y. (2019), "Interview-based research in accounting 2004-2014: informal norms, translations and vibrancy", Management Accounting Research, Vol. 42, pp. 26-38.

Doupnik, T.S. and Richter, M. (2003), "Interpretation of uncertainty expressions: a cross-national study”, Accounting, Organizations and Society, Vol. 28 No. 1, pp. 15-35.

Enzenhofer, E. and Resch, K. (2011), "Übersetzungsprozesse und deren qualitätssicherung in der qualitativen sozialforschung", Forum: Qualitative Sozialforschung, Vol. 12 No. 2, p. 32.

Evans, L. (2004), "Language, translation and the problem of international accounting communication", Accounting, Auditing and Accountability Journal, Vol. 17 No. 2, pp. 210-248.

Evans, L. (2016), "Language, translation and accounting: towards a critical research agenda", Paper presented at the 8th Asia-Pacific Interdisciplinary Research in Accounting Conference, July 2016, RMIT University, Melbourne.

Evans, L. and Kamla, R. (2018), "Language and translation in accounting: a scandal of silence and displacement?", Accounting, Auditing and Accountability Journal, Vol. 31 No. 7, pp. 1834-1843.

Evans, L., Baskerville, R. and Nara, K. (2015), “Colliding worlds: issues relating to language translation in accounting and some lessons from other disciplines", Abacus, Vol. 51 No. 1, pp. 1-36.

Ferri, P. and Zan, L. (2014), “Ten years after: the rise and fall of managerial autonomy in Pompeii”, Critical Perspectives on Accounting, Vol. 25 Nos 4/5, pp. 368-387.

Flamholtz, E.G. (1983), "Accounting, budgeting and control systems in their organizational context: theoretical and empirical perspectives", Accounting, Organizations and Society, Vol. 8 Nos 2/3, pp. 153-169. 
Gambier, Y. (2004), "Translation studies: a succession of paradoxes”, in Schäffner, C. (Ed.), Translation Research and Interpreting Research - Traditions, Gaps and Synergies, Multilingual Matter, Clevedon, pp. 63-70.

Gile, D. (2004), "Response to the invited papers", in Schäffner, C. (Ed.), Translation Research and Interpreting Research - Traditions, Gaps and Synergies, Multilingual Matter, Clevedon, pp. 124-127.

Golden-Biddle, K., Locke, K. and Reay, T. (2006), "Using knowledge in management studies: an investigation of how we cite prior work", Journal of Management Inquiry, Vol. 15 No. 3, pp. 237-254.

González y González, E.M. and Lincoln, Y.S. (2006), "Decolonizing qualitative research: non-traditional reporting forms in the academy", Forum: Qualitative Social Research, Vol. 7 No. 4, Article ID. 1.

Hansson, L. and Longva, F. (2014), "Contracting accountability in network governance structures", Qualitative Research in Accounting and Management, Vol. 11 No. 2, pp. 92-110.

Harkness, J. (2003), “Questionnaire translation”, in Harkness, J., Van de Vijver, F. and Mohler, P. (Eds), Cross-Cultural Survey Methods, Wiley, Hoboken, NJ, pp. 35-56.

Hiebl, M.R.W. (2018), "Management accounting as a political resource for enabling embedded agency", Management Accounting Research, Vol. 38, pp. 22-38.

Holland, J. and Ramazanoglu, C. (1994), "Coming to conclusions: power and interpretation in researching young women's sexuality”, in Maynard, M. and Purvis, J. (Eds), Researching Women's Lives from a Feminist Perspective, Taylor and Francis, London, pp. 125-148.

Hopper, T., Tsamenyi, M., Uddin, S. and Wickramasinghe, D. (2009), "Management accounting in less developed countries: what is known and needs knowing", Accounting, Auditing and Accountability Journal, Vol. 22 No. 3, pp. 469-514.

Hopwood, A.G. (1983), "On trying to study accounting in the context in which it operates", Accounting, Organizations and Society, Vol. 8 Nos 2/3, pp. 287-305.

Houghton, K.A. and Hronsky, J.J.F. (1993), "The sharing of meaning between accounting students and members of the accounting profession", Accounting and Finance, Vol. 33 No. 2, pp. 131-147.

Humphrey, C. and Gendron, Y. (2015), "What is going on? The sustainability of accounting academia", Critical Perspectives on Accounting, Vol. 26, pp. 47-66.

Kamla, R. and Komori, N. (2018), "Diagnosing the translation gap: the politics of translation and the hidden contradiction in interdisciplinary accounting research", Accounting, Auditing and Accountability Journal, Vol. 31 No. 7, pp. 1874-1903.

Kettunen, J. (2017), "Interlingual translation of the international financial reporting standards as institutional work", Accounting, Organizations and Society, Vol. 56, pp. 38-54.

King, N. (2006), "Using interviews in qualitative research", in Cassell, C. and Symon, G. (Eds), Essential Guide to Qualitative Methods in Organizational Research, Sage Publications, Thousand Oaks, CA, pp. 11-22.

Komori, N. (2015), "Beneath the globalization paradox: towards the sustainability of cultural diversity in accounting research", Critical Perspectives on Accounting, Vol. 26, pp. 141-156.

Kruse, J. (2012), "Qualitative interviewforschung im kontext fremder sprachen: eine einleitung", in Kruse, J., Bethmann, S., Niermann, D. and Schieder, D. (Eds), Qualitative Interviewforschung in Und Mit Fremden Sprachen: Eine Einführung in Theorie Und Praxis, Beltz Juventa, Weinheim, pp. 9-26.

Laughlin, R.C. (1988), "Accounting in its social context: an analysis of the accounting systems of the church of England”, Accounting, Auditing and Accountability Journal, Vol. 1 No. 2, pp. $19-42$.

Liyanage, C., Elhag, T., Ballal, T. and Li, Q. (2009), "Knowledge communication and translation - a knowledge transfer model”, Journal of Knowledge Management, Vol. 13 No. 3, pp. 118-131.

Lukka, K. and Kasanen, E. (1995), "The problem of generalizability: anecdotes and evidence in accounting research", Accounting, Auditing and Accountability Journal, Vol. 8 No. 5, pp. 71-90. 
QRAM 17,2

Mcdonald, S. and Hellgren, B. (2009), "The interview in international business research: problems we would rather not talk about", in Marschan-Piekkari, R. and Welch, C. (Eds), Handbook of Qualitative Research Methods for International Business, Edward Elgar, Cheltenham, pp. 264-281.

Marschan-Piekkari, R. and Reis, C. (2009), "Language and translation in cross-cultural interviewing”, in Marschan-Piekkari, R. and Welch, C. (Eds), Handbook of Qualitative Research Methods for International Business, Edward Elgar, Cheltenham, pp. 224-243.

Messner, M., Moll, J. and Strömsten, T. (2017), “Credibility and authenticity in qualitative accounting research", in Hoque, Z., Parker, L.D., Covaleski, M.A. and Haynes, K. (Eds), The Routledge Companion to Qualitative Accounting Research Methods, Routledge, London and New York, NY, pp. $432-443$.

Morin, D. (2011), "Serving as magistrate at the French cour des comptes", Accounting, Auditing and Accountability Journal, Vol. 24 No. 6, pp. 718-750.

Mutiganda, J.C. (2013), "Budgetary governance and accountability in public sector organizations: an institutional and critical realism approach", Critical Perspectives on Accounting, Vol. 24 Nos 7/8, pp. 518-531.

Nassar, M., Al-Khadash, H.A. and Sangster, A. (2011), "The diffusion of activity-based costing in Jordanian industrial companies", Qualitative Research in Accounting and Management, Vol. 8 No. 2, pp. 180-200.

Nida, E.A. (1993), Language, Culture and Translating, Shanghai Foreign Language Education Press, Shanghai.

Nobes, C. (1993), "The true and fair view requirement: impact on and of the fourth directive", Accounting and Business Research, Vol. 24 No. 93, pp. 35-48.

Nobes, C. (2009), "The importance of being fair: an analysis of IFRS regulation and practice - a comment", Accounting and Business Research, Vol. 39 No. 4, pp. 415-427.

Oliver, B.L. (1974), "The semantic differential: a device for measuring the interprofessional communication of selected accounting concepts", Journal of Accounting Research, Vol. 12 No. 2, pp. 299-316.

Parker, L.D. (2012), "Qualitative management accounting research: assessing deliverables and relevance", Critical Perspectives on Accounting, Vol. 23 No. 1, pp. 54-70.

Parker, L.D. and Northcott, D. (2016), "Qualitative generalising in accounting research: concepts and strategies", Accounting, Auditing and Accountability Journal, Vol. 29 No. 6, pp. $1100-1131$.

Pentland, B.T. (1993), "Getting comfortable with the numbers: auditing and the micro-production of macro-order", Accounting, Organizations and Society, Vol. 18 Nos 7/8, pp. 605-620.

Pratt, M.G. (2009), "For the lack of a boilerplate: tips on writing up (and reviewing) qualitative research", Academy of Management Journal, Vol. 52 No. 5, pp. 856-862.

Pugh, M.A. and Vetere, A. (2009), "Lost in translation: an interpretive phenomenological analysis of mental health professionals' experiences of empathy in clinical work with an interpreter", Psychology and Psychotherapy: Theory, Research and Practice, Vol. 82 No. 3, pp. 305-321.

Qu, S.Q. and Dumay, J. (2011), “The qualitative research interview”, Qualitative Research in Accounting and Management, Vol. 8 No. 3, pp. 238-264.

Ramirez, C. (2001), "Understanding social closure in its cultural context: accounting practitioners in France (1920-1939)", Accounting, Organizations and Society, Vol. 26 No. 4/5, pp. 391-418.

Ryan, B., Scapens, R.W. and Theobald, M. (2002), Research Method and Methodology in Finance and Accounting, Cengage Learning, Boston.

Salama-Carr, M. (2009), "Interpretive approaches", in Baker, M. and Saldanha, G. (Eds), Routledge Encyclopedia of Translation Studies, Routledge, London, pp. 145-147. 
Santos, H.P.O., Black, A.M. and Sandolewski, M. (2015), "Timing of translation in cross-language qualitative research", Qualitative Health Research, Vol. 25 No. 1, pp. 134-144.

Santos, H.P.O., Sandolewski, M. and Gualda, D.M.R. (2013b), "Bad thoughts: Brazilian women's responses to mothering while experiencing postnatal depression”, Midwifery, Vol. 30 No. 6, pp. 788-794.

Santos, H.P.O., Gualda, D.M.R., Silveira, M.F.A. and Hall, W. (2013a), "Postpartum depression: the (in) experience of Brazilian primary health care professionals", Journal of Advanced Nursing, Vol. 69 No. 6, pp. 1248-1258.

Non-English interviews in accounting research

Scapens, R.W. (2004), "Doing case study research", in Humphrey, C. and Lee, B. (Eds), The Real Life Guide to Accounting Research, Elsevier, Amsterdam, pp. 257-279.

Schäffner, C. (2004), "Researching translation and interpreting", in Schäffner, C. (Ed.), Translation Research and Interpreting Research - Traditions, Gaps and Synergies, Multilingual Matter, Clevedon, pp. 1-9.

Schäffner, C. (2009), "Functionalist approaches", in Baker, M. and Saldanha, G. (Eds), Routledge Encyclopedia of Translation Studies, Routledge, London, pp. 115-121.

Shapiro, D., Kirkman, B.L. and Courtney, H.G. (2007), "Perceived causes and solutions of the translation problem in management research", Academy of Management Journal, Vol. 50 No. 2, pp. 249-266.

Simon, S. (1997), “Translation, postcolonialism and culural studies”, Meta, Vol. 42 No. 2, pp. 462-477.

Skærbæk, P. (2009), "Public sector auditor identities in making efficiency auditable: the national audit office of Denmark as independent auditor and modernizer", Accounting, Organizations and Society, Vol. 34 No. 8, pp. 971-987.

Skærbæk, P. and Tryggestad, K. (2010), "The role of accounting devices in performing corporate strategy", Accounting, Organizations and Society, Vol. 35 No. 1, pp. 108-124.

Steyaert, C. and Janssens, M. (2012), "Multilingual scholarship and the paradox of translation and language in management and organization studies", Organization, Vol. 20 No. 1, pp. 131-142.

Temple, B. (1997), "Watch your tongue: issues in translation and cross-cultural research", Sociology, Vol. 31 No. 3, pp. 607-618.

Temple, B. (2002), "Crossed wires: interpreter, translators, and bilingual workers in cross-language research", Qualitative Health Research, Vol. 12 No. 6, pp. 844-854.

Temple, B. (2008), "Narrative analysis of written texts: reflexivity in cross language research", Qualitative Research, Vol. 8 No. 3, pp. 355-365.

Temple, B. and Young, A. (2004), "Qualitative research and translation dilemmas", Qualitative Research, Vol. 4 No. 2, pp. 161-178.

Tourish, D. and Willmott, H. (2015), "In defiance of folly: journal rankings, mindless measures and the ABS guide", Critical Perspectives on Accounting, Vol.26, pp. 37-46.

van de Vijver, F. and Tanzer, N.K. (2004), "Bias and equivalence in cross-cultural assessment: an overview", European Review of Applied Psychology, Vol. 54 No. 2, pp. 119-135.

Vinnari, E. and Laine, M. (2013), "Just a passing fad? The diffusion and decline of environmental reporting in the finnish water sector", Accounting, Auditing and Accountability Journal, Vol. 26 No. 7, pp. 1107-1134.

Wade, E. and Clark, H.H. (1993), "Reproduction and demonstration in quotations", Journal of Memory and Language, Vol. 32 No. 6, pp. 805-819.

Wagenhofer, A. (2006), "Management accounting research in German-speaking countries", Journal of Management Accounting Research, Vol. 18 No. 1, pp. 1-19.

Welch, C. and Piekkari, R. (2006), "Crossing language boundaries: qualitative interviewing in international business", Management International Review, Vol. 46 No. 4, pp. 417-437. 
QRAM

17,2

262

\section{Further reading}

Armăsar, I.P. (2014), "Aspects of specialized translations in the field of economics”, Bulletin of the Transilvania University of Braşov, Series V: Economic Sciences, Vol. 7 (56) No. 2, pp. 251-258.

Roberts, J. (2009), "No one is perfect: the limits of transparency and an ethic for 'intelligent' accountability", Accounting, Organizations and Society, Vol. 34 No. 8, pp. 957-970.

\section{Corresponding author}

Martin R.W. Hiebl can be contacted at: martin.hiebl@uni-siegen.de

For instructions on how to order reprints of this article, please visit our website: 